\title{
The School Bus Problem on Trees
}

\author{
Adrian Bock ${ }^{1}$, Elyot Grant ${ }^{2}$, Jochen Könemann ${ }^{2}$, and Laura Sanità ${ }^{1}$ \\ 1 EPFL, Lausanne, Switzerland \\ 2 University of Waterloo, Canada
}

\begin{abstract}
The School Bus Problem is an NP-hard vehicle routing problem in which the goal is to route buses that transport children to a school such that for each child, the distance travelled on the bus does not exceed the shortest distance from the child's home to the school by more than a given regret threshold. Subject to this constraint and bus capacity limit, the goal is to minimize the number of buses required.

In this paper, we give a polynomial time 4-approximation algorithm when the children and school are located at vertices of a fixed tree. As a byproduct of our analysis, we show that the integrality gap of the natural setcover formulation for this problem is also bounded by 4 . We also present a constant approximation for the variant where we have a fixed number of buses to use, and the goal is to minimize the maximum regret.
\end{abstract}

\section{Introduction}

Vehicle routing is an important and active topic in computer science and operations research. In the literature, the objective is typically to find a minimum-cost set of routes in a network that achieve a certain objective subject to a set of constraints. The constraints and cost are often related to the distance travelled, number of routes or vehicles used, coverage of the network by the routes, and so on. Problems of this kind are frequent and crucial in areas such as logistics, distribution systems, and public transportation (see, e.g., the survey by [12]).

In vehicle routing problems relevant to public transportation, a secondary objective often must be taken into account beyond minimizing operation cost: namely, it is crucial to design routes so as to optimize customer satisfaction in order to motivate customers to use the service. This requirement is essential in the so-called School Bus Problem (SBP) - the focus of this paper.

In the SBP, we must route buses that pick up children and bring them from their homes to a school. However, parents do not want their children to spend too much time on the bus relative to the time required to transport them to school by car along a shortest path. In fact, if the additional distance travelled by the bus exceeds a certain regret threshold, the parents would rather drive their children to school by themselves, which is unacceptable. Subject to this, the goal is to cover all of the children using a minimum number of buses.

Formally, we are given an undirected network $G(V, E)$ with distances on the edges $d: E \rightarrow \mathbb{Z}_{+}$, a node $s \in V$ representing the school, and a set $W \subseteq V$ representing the houses of children. Additionally, we are given a bus capacity 
$C \in \mathbb{Z}_{+}$, and a regret bound $R \in \mathbb{Z}_{+}$. The aim is to construct a minimum cardinality set $\mathcal{P}$ of walks ending at $s$ (bus routes) and assign each child $w \in W$ to be the responsibility of some bus $p(w) \in \mathcal{P}$ such that (i) for each walk $P \in \mathcal{P}$, the total number of children $w$ with $p(w)=P$ is at most the capacity $C$; (ii) for every child the regret bound is respected, that is: $d^{P}(w, s) \leq d(w, s)+R$, where $d^{P}(w, s)$ is the distance from the child $w$ to the school $s$ on the walk $p(w)$, and $d(w, s)$ is the shortest distance from $w$ to $s$ in the graph $G$.

In an additional variation of the problem, we have a fixed number $N$ of buses we can use, and the goal is to minimize the maximum regret $R$. We call this variant the School Bus Problem with Regret minimization (SBP-R).

Like many vehicle routing problems, both SBP and SBP-R are strongly NPhard, even on the simplest of graphs. To see this, consider a star centered at the school $s$ with children located at all other vertices. If $k$ of the edges are very long, then determining if $k$ buses are sufficient with a regret bound $R$ is precisely equivalent to solving the bin-packing decision problem with $k$ bins and objects sized according to the distances from the remaining children to the school. It follows that bin-packing can be reduced to both SBP and SBP-R on stars.

Many variants of vehicle routing have been studied in the context of exact, approximate, and heuristic algorithms. For SBP and SBP-R, heuristic methods for practical applications have been examined (see the survey of [10]), but there is no literature concerning formal approximability and inapproximability results. Our goal is to advance the state of the art in this perspective.

To begin, it is easy to see that the SBP can be formulated as a set covering problem. With this observation, one can easily derive a logarithmic approximation as well as a logarithmic upper bound on the integrality gap of the natural set-cover formulation applied to the SBP (see Section 2 for more details). The SBP is closely related (but not equal) to the Distance Constrained Vehicle Routing Problem (DVRP), which is well known and widely studied in terms of approximation. The DVRP can also be approximated within a logarithmic factor in general graphs, but there is a better 2-approximation on trees, as shown by [9]. They also show that the set-cover formulation for the DVRP on trees has integrality gap of at most 20. A natural question is then whether or not the SBP also admits a constant approximation/integrality gap on such graphs. Unfortunately, a straightforward adaptation of their methods to the SBP does not work. Therefore, in order to develop improved approximation results for the $\mathrm{SBP}$, we need to introduce some new ideas.

\subsection{Our results}

We first give a simple combinatorial 4-approximation for the SBP on trees.

Theorem 1. There exists a polynomial time 4-approximation for the SBP on trees. The approximation factor reduces to 3 in the case of unlimited capacity.

In contrast to the results given in 9] for DVRP, our algorithm for SBP immediately yields an integrality gap bound matching the approximation factor: 
Theorem 2. The integrality gap of the natural set-cover formulation of the SBP on trees is at most 4. In case of unlimited capacity, the gap is at most 3.

On the negative side, we can prove an inapproximability factor of $\frac{5}{4}$ for the SBP on trees. Due to lack of space, we refer to the full version of this paper for a proof.

Finally, we give a combinatorial 12.5-approximation for the SBP-R on trees.

Theorem 3. There exists a polynomial time 12.5-approximation algorithm for the $S B P-R$ on trees in the case of unlimited capacity.

\subsection{Related work}

There are an enormous number of results concerning vehicle routing problems; see the survey [12]. The Capacitated Vehicle Routing Problem (CVRP) enforces a limit $C$ on the number of visited locations in each route, and the goal is the minimization of the total length of all the routes. The paper [5] established a strong link to the underlying Travelling Salesman Problem (TSP) by giving an approximation algorithm that relies on the approximation algorithms for TSP. Depending on the capacity bound $C$, it is possible to obtain a PTAS in the euclidean plane for some special cases (if the capacity is either small [1], or very large [2]). If we restrict the input to trees, there is a 2-approximation [6] . Another constraint considered in literature is a bound $D$ on the length of a vehicle tour, under the objective of minimizing the number of routes. This is the Distance Constrained Vehicle Routing Problem (DVRP). It was raised and studied for applications in [7] and [8]. Routing problems like the DVRP can be directly encoded as instances of Minimum Set Cover, and thus often admit logarithmic approximations. The authors of [9] give a careful analysis of the set cover integer programming formulation of the DVRP and bound its integrality gap by $\mathcal{O}(\log D)$ on general graphs and by $\mathcal{O}(1)$ on a tree. They also obtain a constant approximation for the DVRP on a tree and a $\mathcal{O}(\log D)$ approximation in general.

Many practical problems involving school buses have been studied, but primarily within the context of heuristic methods for real-life instances. We refer to [10] for a thorough survey of possible formulations and heuristic solution methods. Our notion of regret was first introduced as a vehicle routing objective in 11]. They considered a more general problem involving timing windows for customers and applied metaheuristics to produce solutions to real-life instances.

\section{Preliminaries}

We first observe that the capacity bound can be neglected for a slight loss in the approximation factor for the SBP. The proof is essentially identical to that of a similar result proved in [9] for the DVRP, and therefore we omit it.

Lemma 1. Given an $\alpha$-approximation to the SBP with unlimited capacity for each bus, there is an $\alpha+1$-approximation to the $S B P$ with capacity bound $C$. 
If $P$ is a walk ending at $s$ and covering a subset $S$ of nodes, then we say that $P$ has regret $R$ if a regret bound of $R$ is respected for all children in $S$. The following useful fact holds for both the SBP and the SBP-R (due to lack of space we omit its easy proof):

Proposition 1. Let $P$ be a walk starting at some node $v$ and ending at $s$. For all nodes covered by $P$ the regret bound $R$ is respected if and only if it is respected for $v$.

We next give a covering integer programming formulation of the SBP. Let $\mathcal{S}$ be the family of all feasible sets of $C$ or fewer children that can be covered by a single walk ending at $s$ having regret at most $R$. We introduce a variable $x_{S}$ for each $S \in \mathcal{S}$ and give the following formulation:

$$
\min \begin{gathered}
\sum_{S \in \mathcal{S}} x_{S} \\
\sum_{S: w \in S} x_{S} \geq 1 \quad \forall w \in W \\
x_{S} \in\{0,1\} \forall S \in \mathcal{S} .
\end{gathered}
$$

An $\mathcal{O}(\log |W|)$-approximation algorithm easily follows from adapting the greedy strategy for set cover. Such a greedy algorithm, applied to an SBP instance, repeatedly searches for a feasible walk ending at $s$ that picks up the maximum number of uncovered children, doing so until every child is picked up. At each iteration, we guess the starting point $v^{*}$ (by trying all $|V|-1$ possibilities). Using Proposition 1 the resulting problem we are left with is to find a $v^{*}-s$ walk in $G$ of length at most $d\left(v^{*}, s\right)+R$ visiting the maximum number of uncovered nodes in $W$. Such a problem is well known in the literature as the Orienteering Problem, and can be approximated within a constant [3, 4]. Following standard methods (e.g., see [13]), we may then obtain an $\mathcal{O}(\log C)$-approximation algorithm for the $\mathrm{SBP}$ and show that the integrality gap of (IP) is at most $\mathcal{O}(\log C$ ) (we refer to the full version of this paper for a rigorous argument). However, these logarithmic results are not known to be tight; in particular, we show how to do better for the SBP on trees.

In the remainder of this paper, we will focus on the infinite capacity version of the SBP on a tree $T$ with root $s$. We denote by $P(u, v)$ and $d(u, v)$ the unique path from $u$ to $v$ in $T$, and its corresponding length. For a subset of edges $F$, we let $d(F):=\sum_{e \in F} d(e)$. An Euler tour of a connected set of edges is a walk that visits each edge exactly twice. We note that subtrees of $T$ that contain no vertices in $W$ will never be visited by a bus in any optimal solution, and thus we can assume without loss of generality that all leaves of $T$ contain children. In such an instance, a feasible solution will simply cover all of $T$ with bus routes and thus is still feasible if every node of $T$ contains a child (assuming infinite capacities). We thus will assume, without loss of generality, that $W=V$.

\section{A 4-approximation to the SBP on trees}

We prove Theorem 1 by first giving a combinatorial 3-approximation for the SBP with unlimited capacity on graphs that are trees, and subsequently applying Lemma 1. Our algorithm is based on the following intuitive observations: 
- When the input tree is very short (say, of height at most $\frac{R}{2}$ on an instance with regret $R$ ), then it is relatively easy to obtain a 2-approximation for the SBP by simply cutting an Euler tour of the tree into short pieces and assigning each piece to a bus.

- General trees can be partitioned into smaller pieces (subtrees) such that at least one bus is required for each piece, but each piece can be solved almost optimally via a similar Euler tour method.

We begin with some definitions. We call a set of vertices $\left\{a_{1}, \ldots, a_{m}\right\} \subseteq V$ $R$-independent if for all $a_{i} \neq a_{j}$, we have $d\left(a_{i}, \operatorname{lca}\left(a_{i}, a_{j}\right)\right)>\frac{R}{2}$, where lca $\left(a_{i}, a_{j}\right)$ is the lowest common ancestor of the vertices $a_{i}$ and $a_{j}$ in $T$. By iteratively marking the leaf in $T$ furthest from the root such that $R$-independence is maintained among marked leaves, we can obtain, in polynomial time, an inclusion-wise maximal $R$-independent set of leaves $A$ such that all vertices in $T$ are within a distance of $\frac{R}{2}$ from a path $P(s, a)$ for some $a \in A$. We shall call $A$ a set of anchors. By construction, no two distinct anchors $a_{i}$ and $a_{j}$ can both be covered by a walk of regret at most $R$, immediately yielding the following lower bound:

Proposition 2. The size $|A|$ of the set of anchors is a lower bound on the number of buses that is needed in any feasible solution.

We now give a second useful lower bound. Let $Q:=\bigcup_{a \in A} P(s, a)$. We call $Q$ the skeleton of $T$, noting that $Q$ is a subtree of $T$ whose leaves are the anchors. Observe that all edges in the skeleton $Q$ will automatically be covered if a bus visits each anchor. Since each anchor must be visited at least once, it suffices to only consider covering the anchors and the non-skeletal edges of $T$, i.e. the edges in $T \backslash Q$. The edges in $T \backslash Q$ form a collection of disjoint subtrees, each of which has height at most $\frac{R}{2}$. We call these short subtrees.

Suppose that a feasible walk starts at a vertex $v$ in a short subtree $\mathcal{T}$. It will cover all the edges in $P(s, v)$, and may possibly cover some additional detour edges having total length at most $\frac{R}{2}$. Since $\mathcal{T}$ is a short subtree, the non-skeletal edges in $P(s, v)$ have total length at most $\frac{R}{2}$. It follows that:

Observation 1 The set of non-skeletal edges covered by any feasible walk $P$ must have total length at most $R$ : at most $\frac{R}{2}$ in length along the path from its starting vertex to the root, and at most $\frac{R}{2}$ length in edges covered by detours.

From this, we can observe the following lower bound on the number of buses:

Proposition 3. The number $\frac{1}{R} \sum_{e \in T \backslash Q} d(e)$ is a lower bound on the number of buses that are needed in any feasible solution.

We build our 3-approximation from these two lower bounds by partitioning the edges of $T$ into a family of subtrees each containing a single anchor, and approximating the optimal solution well on each of these subtrees. For anchors $A=\left\{a_{1}, \ldots, a_{m}\right\}$, we define associated paths of edges $\left\{P_{1}, \ldots, P_{m}\right\}$ as follows: (i) $P_{1}=P\left(s, a_{1}\right)$, and (ii) $P_{i}=P\left(s, a_{i}\right) \backslash\left(\bigcup_{j=1}^{i-1}\left\{P_{j}\right\}\right)$ for $2 \leq i \leq m$. The edges 
in $\left\{P_{1}, \ldots, P_{m}\right\}$ form a partition of the skeleton $Q$ into paths, each of which starts at a different anchor.

We then let $T_{i}$ be the set of all edges in both the path $P_{i}$ and the set of all short subtrees attached to $P_{i}$. If a short subtree is attached to a junction point where two paths $P_{i}$ and $P_{j}$ meet, we arbitrarily assign it to either $P_{i}$ or $P_{j}$ so that the sets $\left\{T_{1}, \ldots, T_{m}\right\}$ form a partition of all of the edges of $T$ into a collection of subtrees, each containing a single anchor.

For each $1 \leq i \leq m$ we define a directed walk $W_{i}$ that starts at the anchor $a_{i}$, proceeds along $P_{i}$ in the direction toward the root $s$, and collects every edge in $T_{i}$ by tracing out an Euler tour around each of the short subtrees in $T_{i}$ that are attached to $P_{i}$. One may easily verify that it is always possible to quickly find such a walk such that the following properties are satisfied:

- $W_{i}$ contains each edge in $P_{i}$ exactly once and always proceeds in the direction toward $s$ when collecting each edge in $P_{i}$.

- $W_{i}$ contains each edge in $T_{i} \backslash P_{i}$ exactly twice: once proceeding in the direction away from $s$, and once in the direction toward $s$.

We now greedily assign the edges in the short subtrees in $T_{i}$ to buses by simply adding edges to buses in the order in which they are visited by $W_{i}$. We first initialize a bus $\beta_{1}$ at the anchor $a_{i}$ and have it travel along $W_{i}$ until the total length of all of the edges it has traversed in the downward direction (away from the root $s$ ) is exactly $\frac{R}{2}$. At this point, we assume it lies on some vertex $v_{1}$ (if not, we may imagine adding $v_{1}$ to the middle of an existing edge in $T_{i}$, although this will not be relevant to our solution as there are then no children at $\left.v_{1}\right)$. We send bus $\beta_{1}$ from $v_{1}$ immediately back to the root $s$ and create a new bus $\beta_{2}$ that starts at $v_{1}$ and continues to follow $W_{i}$ until it too has traversed exactly $\frac{R}{2}$ length in edges of $T_{i}$ in the downward direction. We assume it then lies at a vertex $v_{2}$, create a new bus $\beta_{3}$ that starts at $v_{2}$ and continues to follow $W_{i}$, and so on. Eventually, some bus $\beta_{k}$ will pick up the last remaining children and proceed to the root $s$, possibly with leftover detour to spare. We observe that the number of buses used is exactly $\left\lceil\frac{2 \sum_{e \in T_{i} \backslash P_{i}} d(e)}{R}\right\rceil$ since each bus other than the last one consumes exactly $\frac{R}{2}$ of the downward directed edges in $W_{i}$, and $W_{i}$ proceeds downward along each edge in $T_{i} \backslash P_{i}$ exactly once. We also note that this is a feasible solution since a bus travelling a total downward direction of $\frac{R}{2}$ must make a detour no greater than $R$.

Doing this for each edge set $T_{i}$ yields a feasible solution to the original instance using exactly $\sum_{i=1}^{m}\left\lceil\frac{2 \sum_{e \in T_{i} \backslash P_{i}} d(e)}{R}\right\rceil$ buses. This is at most

$$
m+\frac{2}{R} \sum_{i=1}^{m} \sum_{e \in T_{i} \backslash P_{i}} d(e)=m+2 \frac{\sum_{e \in T \backslash Q} d(e)}{R} \leq 3 O P T
$$

by Proposition 2 and Proposition 3, where $O P T$ is the optimal number of buses required in any feasible solution. Together with Lemma 1 this proves Theorem 1 .

One may notice that the bounds given in Propositions 2 and 3 are necessarily also respected by fractional solutions to the LP relaxation of $(I P)$. Together with 
the argument above, this immediately implies that $(I P)$ has an integrality gap of at most 4 (and 3 in the case of infinite capacities), proving Theorem 2 We refer to the full version of this paper for more details.

\section{A 12.5-approximation to the uncapacitated SBP-R on trees}

In this section, the School Bus Problem with Regret Minimization (SBP-R) is considered. In SBP-R, the number of routes is bounded by a given parameter $N \in \mathbb{N}$ while the maximum regret is to be minimized. We prove Theorem 3 by giving a polynomial time 12.5-approximation algorithm for SBP-R.

Without loss of generality, we may assume the tree $T$ to be binary. Suppose we can fix a value $R$ for the regret. We will develop an algorithm that, given an instance and the value $R$, either outputs a set of at most $N$ bus routes, with a maximum regret of $12.5 R$, or asserts that every solution with at most $N$ buses must have a regret value $>R$. Then, we can do binary search on the regret values and output the best solution found.

Suppose we have guessed a value for $R$. First, we find a set of anchors $A$ with respect to $R$ as described in section 3. Now, we look for a set of routes which only start at the anchors. Using the notion introduced for SBP in section 3. our strategy is to cluster and cut the short subtrees into suitable pieces (called tickets) that can be collected efficiently by buses. In order to explain the cutting technique for the short subtrees, we need to introduce some more definitions.

Every vertex $v \in Q$ of the skeleton is called a junction point if either it is the root $s$ or $v$ has degree more than 2 in $Q$. Let $J$ be the set of junction points. The skeleton $Q$ can be split at its junction points into a set of edge-disjoint paths, which we will call core segments. Formally, a path in $Q$ is a core segment if and only if its endpoints are anchors or junction points and it contains no junction points in its interior. The next lemma shows how we obtain suitable tickets from a collection of short subtrees.

Lemma 2. There exists a polynomial-time algorithm that, given a path $P$ and a collection $\mathcal{C}$ of short subtrees whose roots lie on $P$, produces a partition of the edges of $\mathcal{C}$ into tickets $E_{0}^{\mathcal{C}}, E_{1}^{\mathcal{C}}, \ldots, E_{k}^{\mathcal{C}}$ with $k \leq\left\lfloor\frac{\sum_{\mathcal{T} \in \mathcal{C}} d(\mathcal{T})}{R}\right\rfloor$ such that:

(P1) All of the edges in $E_{0}^{\mathcal{C}}$ can be collected with an additional regret $\leq 2.5 R$ by a single bus whose route contains $P$.

(P2) For all $1 \leq i \leq k$, all the edges in $E_{i}^{\mathcal{C}}$ can be collected with an additional regret at most $3 R$ by a single bus whose route contains $P$.

Proof. Since tree $T$ is binary it follows that each short subtree in $\mathcal{C}$ has a single root edge that connects it to the skeleton $Q$. Shift each of the short subtrees to the lowest node $v$ of path $P$, and subsequently compute the Euler tour $\tau$ of all short subtrees with root $v$. Tour $\tau$ has the following important contiguity property: the edges of each of the short trees in $\mathcal{C}$ form a contiguous sub-path. 
We now cut $\tau$ into suitable pieces: Starting at $v$, walk along $\tau$, and cut it at the first node such that the resulting piece has length $>2 R$. Continue like this to obtain $k+1$ tickets. Denote by $E_{1}^{\mathcal{C}}, \ldots, E_{k}^{\mathcal{C}}$ all but the last piece. The last part of the Euler tour defines $E_{0}^{\mathcal{C}}$. Note that $k \leq\left\lfloor\frac{\sum_{\mathcal{T} \in \mathcal{C}} d(\mathcal{T})}{R}\right\rfloor$, and that every cutting point is covered by exactly two tickets. Since every node needs to be covered only once in a solution, we can remove the last edge from each set $E_{i}^{\mathcal{C}}$ $(1 \leq i \leq k)$; note that the resulting ticket has length at most $2 R$.

The construction together with the contiguity property of $\tau$ implies that the walk corresponding to $E_{i}^{\mathcal{C}}$ partially visits at most two short subtrees (at the beginning and end); all other short subtrees are either fully contained in $E_{i}^{\mathcal{C}}$, or not at all. Start and end vertex of the ticket are also at distance at most $\frac{R}{2}$ from $v$ (cf. the definition of a short subtree); it follows that a bus whose root walk contains $P$ can cover all edges corresponding to a ticket with regret at most $3 R$.

The last piece $E_{0}^{\mathcal{C}}$ of the Euler tour remaining from the cutting procedure certainly has length $\leq 2 R$. Since the Euler tour goes back to $v$, only the distance to the starting point of the last piece has to be connected to $v$ in order to obtain a set of tours. As in the previous case, one bus can cover these tours with regret $2.5 R$, since the height of each subtree is at most $\frac{R}{2}$.

This strategy fulfills the properties (P1) and (P2) and runs in polynomial time.

The next simple lemma (whose proof is omitted) will be useful later.

Lemma 3. One can find a mapping $\phi: J \longrightarrow A$ from junction points to anchors in polynomial time with the following properties:

(i) For all $j \in J$, the junction point $j$ lies on the path $P(s, \phi(j))$;

(ii) For all $a \in A$, there is at most one junction point $j \in J$ with $\phi(j)=a$.

For every core segment $S$, let $t(S)$ and $b(S)$ be the top and the bottom junction points in $S$. Let further $r(S)$ be the highest junction point at distance at most $R / 2$ from $t(S)$ (see Fig. at side). Our algorithm works as follows.

\section{Algorithm 1}

1. Find a maximal $R$-independent set of anchors $A$.

2. Initialize a default bus at each anchor $a \in A$.

3. For each junction point $j \in J$ in bottom-up order do:

Assign an arbitrary left-to-right ordering of the two segments $S_{l}, S_{r}$ with $t\left(S_{l}\right)=j=t\left(S_{r}\right)$.

(a) Let $\mathcal{C}_{1}$ be the collection of short subtrees whose root node lies in the left core segment $S_{l}$ at distance $\leq R / 2$ from $j$. Let $\mathcal{C}_{2}$ be the collection of short subtrees whose root node lies in the core segment $S_{j}$ with $b\left(S_{j}\right)=j$ at distance $>R / 2$ from $t\left(S_{j}\right)$. Note that $C_{2}=\emptyset$ is possible.

(b) Apply Lemma 2 to $\mathcal{C}_{1} \cup \mathcal{C}_{2}$ on $P=S_{l} \cup S_{j}$, and obtain tickets $E_{0}, E_{1}, \ldots, E_{y}$.

(c) Let $\mathcal{C}_{3}$ be the collection of short subtrees whose root node lies in the right core segment $S_{r}$ at distance $\leq R / 2$ from $j$.

(d) Apply Lemma2 2 to $\mathcal{C}_{3}$ on path $P=S_{r}$, and obtain tickets $F_{0}, F_{1}, \ldots, F_{z}$.

(e) Assign the tickets $E_{0}, F_{0}$ to the default bus at $\phi(j)$; remove these tickets.

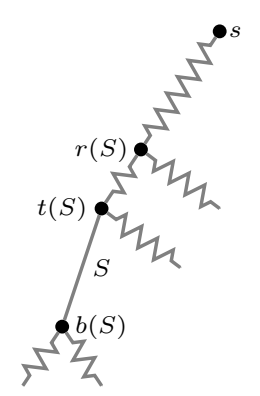


(f) Place the $y$ tickets $E_{1}, \ldots, E_{y}$ and $z$ tickets $F_{1}, \ldots, F_{z}$ at $r\left(S_{l}\right)=r\left(S_{r}\right)$. 4. For each anchor $a \in A$ do:

Let $\mathcal{C}_{a}$ be the collection of short subtrees whose root node lies in the core segment $S_{a}$ with $b\left(S_{a}\right)=a$ at distance $>R / 2$ from $t\left(S_{a}\right)$.

(a) Apply Lemma 2 to $\mathcal{C}_{a}$ on $P=S_{a}$, and obtain tickets $K_{0}, K_{1}, \ldots, K_{w}$.

(b) Assign $K_{0}$ to the default bus at $a$ and remove this ticket.

(c) Place the $w$ tickets $K_{1}, \ldots, K_{w}$ at $a$.

5. Assign every bus greedily the lowest ticket available on its path to the root.

6. Add a new bus for each unmatched ticket.

Lemma 4. Algorithm 1 outputs a set of buses with maximum regret $12.5 R$.

Proof. Any bus starting at an anchor $a$ collects at most 4 different regret amounts:

- at most one ticket from the matching in step 5. In the worst case, the ticket is placed at a junction point $r(S)$ where $S$ denotes the segment where the subtrees contributing to the ticket are rooted. The top junction point $t(S)$ is at distance at most $\frac{R}{2}$ from $r(S)$, by definition. Since the subtrees considered for the ticket are rooted on $S$ at distance at most $\frac{R}{2}$ from $t(S)$, every tour of a ticket is rooted at the skeleton at distance $\leq R$ from $P(s, a)$. Together with $(P 2)$, we can cover a ticket with a walk of regret $\leq 5 R$.

- at most two remaining pieces of the Euler tour in step 3(e). There is at most one junction point $j$ with $\phi(j)=a$ by (ii) of lemma 3. For a junction point $j$, each part $E_{0}$ and $F_{0}$ can be covered with regret $\leq 2.5 R$ by $(\mathrm{P} 2)$.

- at most one remaining piece of the Euler tour at $a$ assigned in step 4(b). This ticket $K_{0}$ can be covered with regret $\leq 2.5 R$ by $(\mathrm{P} 2)$.

In total, the bus from anchor $a$ collects regret of at most $5 R+5 R+2.5 R=12.5 R$.

The following observation (proof omitted) is used to prove the next lemma.

Observation 2 Any edge $e \in T \backslash Q$ that is contained in a ticket $\mathcal{T}$ placed at a junction node $j$ must be covered by a bus starting from an anchor in the subtree rooted at $j$.

Also if $A$ is a set of anchors in $T$, then $A \cap F$ is a set of anchors of any subtree $F \subseteq T$. Let $B$ be the number of bus routes output by the algorithm. We obtain:

Lemma 5. $B$ is a lower bound on the number of buses with regret at most $R$ needed to cover all points.

Proof. Observe that to cover all points, the number of arbitrary buses with regret $\leq R$ is at least the minimum number of buses from anchors that cover $\leq R$ nonskeletal edges each. To show that $B$ is a lower bound on the latter number, we apply Observation 11 and 2 locally and show how to combine the lower bounds from two disjoint subtrees. We can interpret $B$ as the number of anchors plus the number of tickets that are not assigned to a bus in step 5 of the algorithm. Note that at every anchor requires a bus by Proposition 2 . We traverse the tree now bottom up from the anchors and inductively compute at each anchor or 
junction point a lower bound on the number of buses. We distinguish two cases at a node $j$ that is either an anchor or a junction point:

Case 1: There is no unmatched ticket placed at $j$. If $j$ is an anchor, Proposition 2 applies. Otherwise, since all tickets at $j$ are matched, we can focus on the lower bounds at the two successor junction points $j_{1}, j_{2}$ below $j$. It follows from Observation 2 that the lower bound at $j$ is the sum of the buses needed at each of the disjoint subtrees rooted at $j_{1}$ and $j_{2}$.

Case 2: There is at least one unmatched ticket placed at $j$. Due to Observation 2 we look at the subtree below $j$. By step 5 of the algorithm, every bus starting in this subtree is full. Thus Observation 1 proves that we need an additional bus for each ticket. This yields that the lower bound is the sum of the number of buses used below $j$ plus the number of unmatched tickets.

\section{References}

[1] Adamaszek, A., Czumaj, A., and Lingas, A. (2009) PTAS for $k$-Tour Cover Problem on the Plane for Moderately Large Values of $k$. Algorithms and Computation, LNCS 5878, pp. 994-1003, Springer, 2009.

[2] Asano, Tetsuo and Katoh, Naoki and Tamaki, Hisao and Tokuyama, Takeshi (1997): Covering points in the plane by k-tours: towards a polynomial time approximation scheme for general k. STOC 1997.

[3] Blum, A., Chawla, S., Karger, D. R., Lane, T., Meyerson, A., and Minkoff, M. (2007): Approximation Algorithms for Orienteering and Discounted-Reward TSP. SIAM Journal on Computing, vol. 37, no. 2, pp. 653-670, 2007.

[4] Chekuri, C., Korula, N., and Pal, M. (2008) Improved Algorithms for Orienteering and Related Problems. in SODA, pp. 661-670, 2008.

[5] Haimovich, M., and Rinnoy Kan, A.H.G. (1985) Bounds and heuristic for capacitated routing problems. Mathematics of OR, 10(4), pp. 527-542, 1985.

[6] Labbe, M., Laporte, G., and Mercure, H. (1991) Capacitated Vehicle Routing on Trees. Operations Research, vol. 39(4), pp. 616-622, 1991

[7] Laporte, G., Desrochers, M., and Norbert, Y. (1984): Two exact algorithms for the Distance Constrained Vehicle Routing Problem. Networks, vol. 14, pp. $47-61,1984$.

[8] Li, C.-L., Simchi-Levi, S., and Desrochers, M. (1992): On the distance constrained vehicle routing problem. Operations Research, vol. 40, pp. 790-799, 1992.

[9] Nagarajan, V., and Ravi, R. (2008): Approximation Algorithms for Distance Constrained Vehicle Routing Problems. Tepper School of Business, Carnegie Mellon University, Pittsburgh 2008.

[10] Park, J., and Kim, B.-I. (2010): The school bus routing problem: A review. European Journal of Operational Research, vol. 202, pp. 311-319, 2010.

[11] Spada, M., Bierlaire, M., and Liebling, Th. M. (2005): Decision-Aiding Methodology for the School Bus Routing and Scheduling Problem. Transportation Science, vol. 39(4), pp. $477-490,2005$.

[12] Toth, P., and Vigo, D. (2001): The Vehicle Routing Problem. 2001.

[13] Vazirani, V. V. (2001): Approximation Algorithms. Springer, 2001. 